\title{
Radiographic Assessment of Impacted Canine: A
} Systematic Review

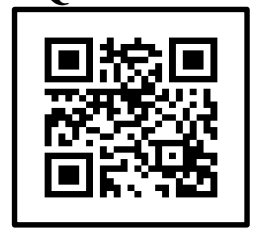

\section{POORNIMA R ${ }^{1}$, N KANNAN ${ }^{2}$, R KRISHNARAJ33, R KAMAL KANTHAN 4 , M DEIVANAYAGI5}

Dental professionals face a lot of challenges in treatment of impacted canine due its position. Localisation of impacted canine in diagnosis and treatment is important. There are various radiographic methods in localization of impacted canine. In this article, different radiographic methods in the diagnosis of impacted canine. The use of periapical radiograph, panoramic radiograph, occlusal radiograph, CT scan, and CBCT have been reviewed using various literature. CBCT gives an accurate dimension and position of impacted canine.

KEYWORDS: Impacted canine, Radiograph, CBCT, CT Scan

\section{INTRODUTION}

Impacted maxillary canine is a common challenge faced by orthodontist in day to day practice. With early detection and proper guidance of impacted teeth we can avoid the deleterious effect of the impacted teeth. In this article we highlight the radiographic techniques that are useful in diagnosis of impacted canine. The radiographic assessment of impacted canines has two important targets 1) qualitative information regarding normal and abnormal position of the teeth 2) position of canine in different planes of space.

\section{MATERIALS AND METHODS}

A search was performed using PubMed, Google Scholar, Science Direct from 1980. The search strategy included terms like radiographic assessment of impacted teeth, diagnosis of impacted canine, canine impaction radiographic illustration. The aim was to identify all papers dealing with radiographic assessment impacted teeth. Criteria for this review were case reports and comparative studies. The reference list of the article were pursued and the articles with related reference were also evaluated.

\section{Inclusion Criteria:}

1) Studies related to radiology of impacted teeth.

2) Studies that have clear description of materials and method.

3) Studies with adequate biostatistics.

\section{Exclusion Criteria:}

1) Abstracts

2) Author debates

3) Syndromes

\section{RESULTS}

Eighteen relevant studies were identified, which comprised of 3 retrospective studies, 5 prospective studies, 6 experimental studies, 2 review articles and 2 case reports. The article selection has been illustrated in PRISMA table (Figure 1).

\section{EFFECTS OF INTERVENTION}

\section{a. Periapical Radiograph}

Periapical radiograph gives information regarding stage of eruption, size of follicle, root resorption. Periapical radiograph is a two dimensional view which cannot give information on buccolingual plane. Erickson et al. ${ }^{1}$ concluded that by individualizing the radiologic investigation according to a stepwise program, it is possible to minimize the total radiation exposure without losing essential information. In cases that involve aberrant eruption of the canines, radiologic examination can be limited to 2 or 3 intraoral films exposed in different projections providing reliable information on the position and eruption pathway(s) of the canines among most children. Determination of diagnostic problems caused by overlapping and interrupted lamina dura and the risk of resorption shall call for the use of 
supplementary investigations. ${ }^{1}$

\section{b. Occlusal Radiograph}

Occlusal radiograph is a true occlusal view and it gives all information of posterior teeth in cross section and also gives information on buccolingual position of canine. Carol Mason et al. ${ }^{2}$ concluded that localization of impacted canine is more successful in occlusal radiograph. He also stated that anterior occlusal view is required in case of buccally placed canine which cannot be palpated.

\section{c. Panoramic Radiograph}

Panoramic radiograph gives good view of all the teeth, jaws and TMJ. According to Nagpal A et al., panoramic radiograph gives prediction of palatal canine in $80 \%$ of patients, but it cannot be used as a sole radiograph for diagnosis and can be used as an adjunct in diagnosis of impacted canine. ${ }^{3}$ Enrique Fernandez et al. suggested that overlapping canine and lateral incisor in panoramic radiographs after the root completion in incisor may be a sign of eruptive disorders of the canine, suggesting for preventive measures to avoid impaction. ${ }^{4}$ So, panaoramic radiograph also helps in prevention of canine impaction by early diagnosis. Gavel et al. concluded and gave six points for identification of impacted canine in panoramic view, which were: 1) the more the tooth is upright in the frontal plane, the more the inclination of the tooth in sagital plane. 2) palatal impactions widens the crown width in comparison to the canine on the opposite side. 3) the impacted position of the tooth does not influence the angulation of the tooth in occlusal plane. 4) buccal and median impaction shortens the distance to the midsagittal plane ,palatal impaction widens the distance 5) palatally impacted canine projects the crown point higher than buccally impacted canine.6)increased curvature of the root demonstrates the palatal impaction of the canine. ${ }^{5}$ Fox et al. reported that if a canine is relatively magnified when compared to adjacent teeth in the arch or contra lateral canine, it will be closer to the tube that is palatal and if it is diminished it is away from the tube that is buccal. ${ }^{6}$

\section{d. Parallax method}

Parallax method is the most common method used for localizing unerupted canines. It can be either horizontal or vertical parallax method. In horizontal parallax method two intra oral films are used and vertical parallax method anterior occlusal films are used. Armstrong et al. concluded that horizontal parallex is more significant than vertical parallex method. $69 \%$ of palatal impaction of maxillary canine were identified with vertical parallex method where as $88 \%$ was identified with horizontal parallex method. ${ }^{7} \mathrm{He}$ also concluded that accuracy in vertical parallex method is less than horizontal parallex method. Jacob et al. have recommended a modification of angulation in vertical parallex method that the angles should be changed from 60 to 65 and 70 to $75 .^{8}$

\section{e. Computerized Tomography (CT)}

In 1988 importance of CT scanning was proposed by Erickson et al. to assess the root resorption. ${ }^{9} \mathrm{He}$ also used CT scanning to identify the position of palatally impacted canine. ${ }^{7}$ Bodner et al. concluded that CT helps in assessing the proximity of impacted tooth to an adjacent pathological lesion. ${ }^{10}$ Sawamura $\mathrm{T}$ et al. have evaluated the use of $3 \mathrm{D} C \mathrm{CT}$ to assess impacted canine and of impacted canine is clear but they also advocated that the use of $3 \mathrm{D} C \mathrm{CT}$ should be done with caution due to radiation exposure. ${ }^{11}$

\section{f. Cone Beam Computerized Tomography (CBCT)}

Merett et al. have concluded with few case reports that $\mathrm{CBCT}$ helps the clinician to get a clear picture on position of impacted teeth accurately. ${ }^{12}$ Walker et al. in their study concluded that CBCT has given an accurate picture on position and volumetric analysis of impacted canine. They also concluded that the images of CBCT give accurate value. ${ }^{13}$ Caroline $S$ et al. analysed the location of impacted maxillary canines and factors that influence root resorptions(s) of adjacent teeth using Cone-Beam Computed Tomography (CBCT) techique. They concluded that $\mathrm{CBCT}$ provides information that is accurate regarding the location of impacted canine and information on root resorption of adjacent teeth. ${ }^{14}$

\section{DISCUSSION}

Most of the studies give information of different radiological techniques to assess the position of impacted canine but periapical radiograph is a $2 \mathrm{D}$ image which gives minimal information. Panoramic view gives more information on 
impacted teeth. Position of the impacted canine, number, location, and amount of resorptions on adjacent teeth, and information on cystic formation of the dental follicle of the canine were identified with help of periapical radiograph. Orthopantograpgh gives information on position of the impacted and adjacent teeth. Dental-CT images are useful for evaluating the shape of the root of an impacted tooth in the maxilla. Because of the local radiation dose from CT is large, proper judgment based on sufficient consideration of the advantages and disadvantages of CT is needed before performing CT examination of impacted teeth. Cone beam computerized tomography gives accurate information of position of the impacted teeth. CBCT imaging is a precise technique in determining not only the labial/lingual relationship of teeth, but also provides details about the exact angulation of the impacted canine(s). These $3 \mathrm{D}$ images generated are beneficial in determining the proximity of the roots of adjacent incisor and premolar teeth, which can be of great value in determining the ease of uncovering and bonding and the vector of force(s) applied that shall be used to move the tooth into the arch with a decreased chance of root resorption of the adjacent tooth.

\section{CONCLUSION}

This literature reveals that $\mathrm{CBCT}$ gives more precise information on position, angulations and relationship with adjacent structures of impacted teeth. OPG also gives information on position and number of impacted teeth. Periapical radiograph gives minimal information and more information like cystic lesion around the impacted teeth.

\section{REFERENCES}

1. Erikson S, Kurol J. Radiographic Examination of ectopically erupting maxillary canines. Am J Orthod Dentofacial Orthop 1987;91(6):493-92.

2. Mason C, Papadakou P, Roberts GJ.. The radiographic localization of impacted maxillary canine: a comparison of methods. Eur J Orthod 2001;23(1):25-34.

3. Nagpal A, Pai KM, Shetty S, Sharma G. Localization of impacted maxillary canines using panaromic radiography. J Oral Sci 2009;51(1):37-45.
4. Ferndndez E, Bravo ALA, Canteras M. Eruption of the permanent study upper canine: A radiologic study. J Orthod Dentofacial Orthop 1998;113(4):414-20.

5. Gavel V, Dermaut L. The effect of tooth position on the image of Unerupted canines on panaoramic radiograph. Eur J Orthod 1999 ;21(5):551-6o.

6. Fox NA, Fletcher GA, Horner K. Localising maxillary canines using dental panoramic tomography. Br Dent J 1995 ;179(11-12):416-20.

7. Armstrong C, Jhonston C, Burden D, Stevanson M. Localizing ectopic maxillary canines-horizontal or vertical parallax?. Eur J Orthod 2003;25(6):585-9.

8. Jacob SG. Localization of maxillary canine: how to and when. Am J Orthod Dentofacial Orthop 1999;115(3);314-22.

9. Ericson S, Kurol J. CT diagnosis of ectopically erupting maxillary canines-a case report. Eur J Orthod 1988;10(2):115-21.

10. Bodner L, Bar-ziv J, Becker A. Image accuracy of plain film radiography and computerized tomography in assessing morphological abnormality of impacted teeth. Am J Orthod Dentofacial Orthop $2001 ; 120(6): 623-8$.

11.Sawamura T, Minowa K, Nakamura M. Impacted teeth in the maxilla: usefulness of 3D Dental-CT for preoperative evaluation. Eur J Radiol 2003;47(3):221-6.

12. Merrett SJ, Drage NA, Durning P. Cone beam computed tomography: a useful tool in orthodontic diagnosis and treatment planning. J Orthod 2009;36(3):202-10.

13. Walker L, Enciso R, Mah J. Three-dimensional localization of maxillary canines with conebeam computed tomography. Am J Orthod Dentofacial Orthop 2005;128(4):418-23.

14. Lai CS, Bornstein MM, Mock L, Heuberger BM, Dietrich T, Katsaros C. Impacted maxillary canines and root resorptions of neighbouring teeth: a radiographic analysis using cone-beam computed tomography. Eur J Orthod 2013;35(4):529-38. 


\section{Cite this article as:}

Poornima R, Kannan N, Krishnaraj R, Kanthan RK, Deivanayagi M. Radiographic Assessment of Impacted Canine: A Systematic Review. Int Healthcare Res J 2018;1(10):304-308.

AUTHOR AFFILIATIONS:

1. Reader, Department of Orthodontics, SRM Dental College, Ramapuram, Chennai-86

2. MDS (Orthodontics), Consultant Dental Orthodontist

3. Professor, Department of Orthodontics, SRM Dental College, Ramapuram, Chennai-86

4. MDS (Oral and Maxillofacial Surgery), Associate Consultant, International Medical Center, Jeddah, Saudi Arabia

5. Oral Medicine and Radiology, Senior Lecturer, Ragas Dental College, Uthandi

\section{Corresponding Author: \\ Dr. N Kannan \\ Consultant Orthodontist \\ Sri Solai Dental Care \\ CP-23, Razack Garden Main Road MMDA Colony Arumbakam \\ Chennai -106}

For article enquiry/author contact details, e-mail at: manuscriptenquiry.ihrj@gmail.com 


\section{LEGEND}

\section{PRISMA 2009 Flow Diagram}
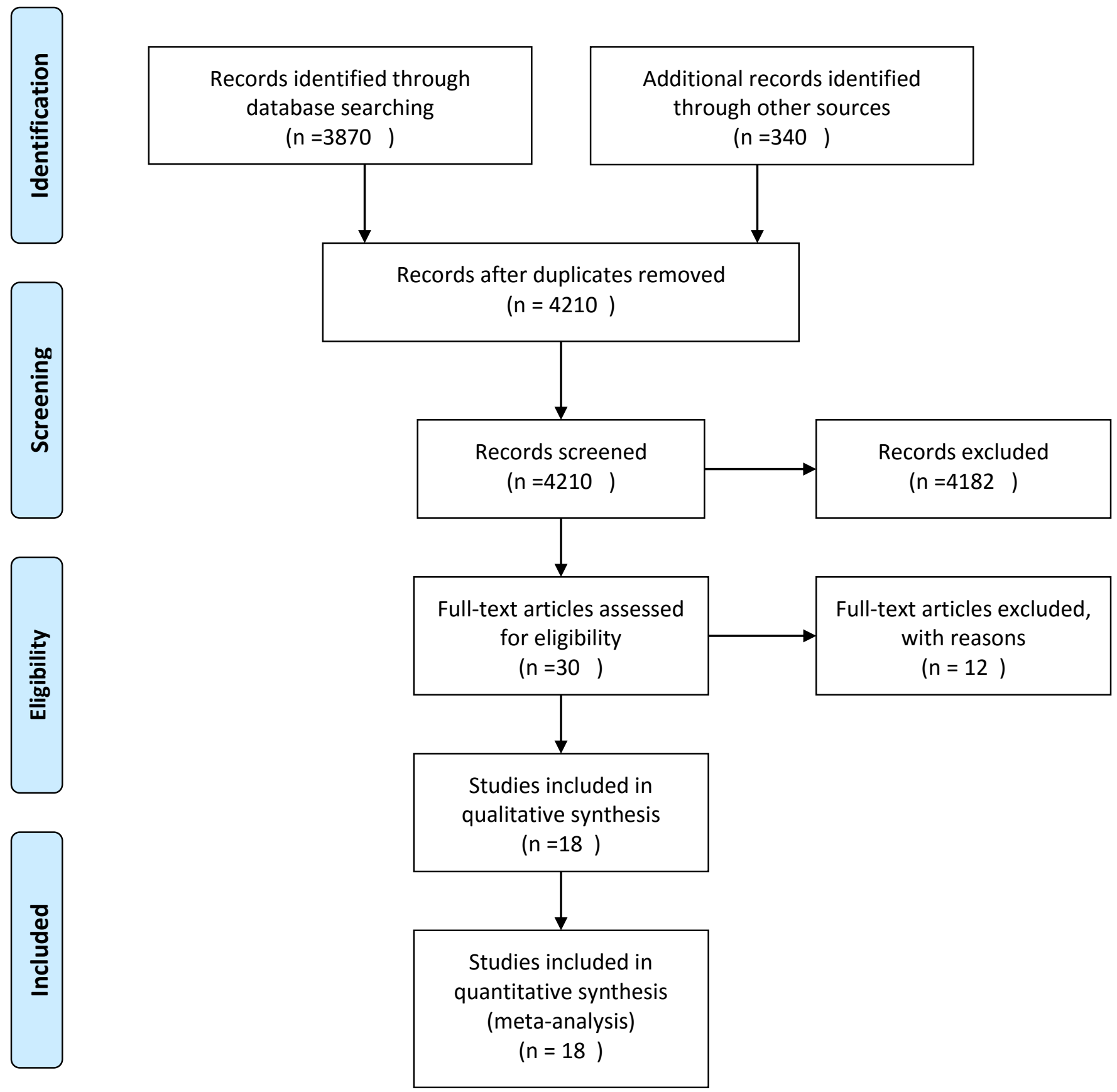